\title{
Acute Pancreatitis in a Pregnant Women at 30 - 31 Weeks of Gestational Age with Complete Cure
}

\author{
Farahnaz Farzaneh (iD ${ }^{1,}{ }^{*}$, Arezoo Esmaeilzadeh ${ }^{2}$ and Raheleh Rezaei ${ }^{2}$ \\ ${ }^{1}$ Infectious Disease and Tropical Medicine Research Center, Zahedan University of Medical Sciences, Zahedan, Iran \\ ${ }^{2}$ Zahedan University of Medical Sciences, Zahedan, Iran \\ "Corresponding author: Infectious Disease and Tropical Medicine Research Center, Zahedan University of Medical Sciences, Zahedan, Iran. Email: farahnaz1826@yahoo.com
}

Received 2019 March 09; Revised 2019 April 19; Accepted 2019 May 13.

\begin{abstract}
Introduction: Acute pancreatitis is an acute inflammatory disease of the pancreas characterized clinically by upper quadrant pain and elevated levels of enzymes in the blood. Although The pathogenesis of pancreatitis is not fully understood, gallstone and chronic alcohol abuse is considered for two-thirds or more cases in the united stated.

Case Presentation: In this case report, the researchers present a 29-year-old pregnant female G3P2 with 31 w, $2 \mathrm{~d}$ of gestational age, who was referring to maternity ward with upper quadrant pain, nausea, and vomiting. Her ultrasound, examination, and blood analysis showed acute pancreatitis due to hyperlipidemia. The patient underwent six plasmapheresis and medical treatment and was discharged with complete cure at 34 weeks of gestational age.

Conclusions: Accurate assessment of the incidence and mortality of acute pancreatitis is difficult as mild pancreatitis may be subclinical and deaths may occur before the diagnosis of sever and fulminant attacks. Mortality rate is three percent in patients with interstitial pancreatitis and $17 \%$ in patients with pancreatic necrosis.
\end{abstract}

Keywords: Acute Pancreatitis, Pregnancy, Triglyceridemia, Plasmapheresis

\section{Introduction}

Pancreatitis is inflammation in the pancreas. The pancreas is a long flat gland that is located in the upper abdomen. It produces enzymes and hormones that help digestion and regulate the glucose processes.

Acute pancreatitis can be divided to two broad categories based on Atlanta classification (1).

-Interstitial edematous acute pancreatitis is characterized by acute inflammation of the pancreatic parenchyma and per pancreatic tissues without recognizable tissue necrosis.

Acute pancreatitis according to severity is divided to the following:

-Mild acute pancreatitis, which is characterized by the absence of organ failure or transient organ failure (lesser than 48 hours) and/or local complications.

-Sever acute pancreatitis, which is characterized by persistent organ failure (greater than 48 hours) that may involve one or multiple organs.

At initial evaluation, the severity of acute pancreatitis should be assessed by clinical examination to assess for early fluid losses, organ failure (particularly cardiovascular, respiratory or renal compromise) measurement of the APACHE2 score, and systematic inflammatory response syndrome (SIRS) score $(1,2)$. Physical findings vary depending upon the severity of acute pancreatitis. In patients with mild acute pancreatitis, the epigastrium may be minimally painful in palpation. In contrast, in patients with severe pancreatitis, there may be significant tenderness to palpation in the epigastrium.

Patients may have abdominal distention and hypoactive bowel sounds (BS) due to an ileus secondary to inflammation. Patients with severe pancreatitis may have hypoxemia, fever, tachypnea, and hypotension.

In three percent of patients with acute pancreatitis, ecchymosis may be in the periumbilicus (Cullen sign) or the flank (grey turner sign). Patients with severe acute pancreatitis may have dyspnea due to diaphragmatic inflammation secondary to pancreatitis, pleural effusions, or adult respiratory distress syndrome.

Approximately five to ten percent of patients with acute severe pancreatitis may have painless disease and have unexplained hypotension (e.g. dialysis and organophosphate poisoning). Although measurement of lipase and amylase is useful for diagnosis of pancreatitis, serial measurements in patients with acute pancreatic 
are not useful to predict disease prognosis and severity or for changing the management. Serum amylase rises within 6 to 12 hours of the onset of acute pancreatitis. Amylase has a short half-life of approximately 10 hours and in uncomplicated attacks returns to normal within three to five days. Serum amylase elevation of greater than three times the upper limit of normal has a sensitivity for the diagnosis of acute pancreatitis of 67 to 83 percent and a specificity of 85 to 98 percent. Serum lipase has a sensitivity and specificity for acute pancreatitis ranging from 82 to 100 percent. Serum lipase rises within four to eight hours of the onset of symptoms, peaks at 24 hours, and returns to normal within 8 to 14 days. Lipase elevations occur earlier and last longer as compared with elevations in amylase and are therefore especially useful in patients, who present greater than 24 hours after the onset of pain. Serum lipase is also more sensitive as compared with amylase in patients with pancreatic secondary to alcohol, after the onset of acute pancreatitis (3).

Overall acute pancreatitis is rare in pregnancy, occurring most commonly in the third trimester, and gallstones are the most common cause. When laparoscopic cholecystectomy is not feasible and a common bile duct stone is highly suspected on imaging, endoscopic sphincterotomy or stenting may help prevent recurrence and postpone cholecystectomy until after delivery (Table 1).

\section{Case Presentation}

In this case report, a 29-year-old pregnant female G3P2 (Gravida 3, Para 2) with gestational age of 31 weeks and three days referred to the maternal ward. Symptoms of this patient were epigastrium pain, RUQ pain, nausea, and vomiting without any delivery (labor pain, PROM, vaginal bleeding) or others sever signs. The vital signs were normal, fetal heart rate was 155 beat per minute and fundal height was normal. The patient did not utilize alcohol or cigarette.

Past medical history (PMH) of the patient showed hyper-lipidemia and gestational diabetic mellitus; she was on a diet for GDM. In her drug history, cholestyramine was prescribed from three days before admission due to rising TG. After admission, the author ordered NPO, N/serum, NGtube, echocardiography, and surgery consultation. Blood Analysis (LFT, Amylase, Lipase, Bun, Cr, CBC, CRP, lipid profile, BS, Na, K, U/A) and abdominal-pregnancy ultrasound was requested

The initial blood analysis showed Amylase $=131, \mathrm{CRP}=$ 96, total cholesterol $=1078, \mathrm{TG}=6036, \mathrm{LFT}=\mathrm{Normal}, \mathrm{Cr}, \mathrm{Bun}$ $=\mathrm{NL}, \mathrm{CBC}(\mathrm{WBC}=14500, \mathrm{HB}=10, \mathrm{PLT}=214000) \mathrm{BS}=177, \mathrm{Cr}$ $=0.9, \mathrm{Na}=13, \mathrm{~K}=4, \mathrm{ALT}=14, \mathrm{AST}=17, \mathrm{LDH}=207$, Albumin
$=3.8$, total bitt $=2.5$, direct bill $=1.4$ and viral marker was normal. Echocardiography showed Nl EF and PAP.

The ultrasound showed multi-locolar collection in pancreas with normal gall bladder and liver. Her pregnancy was 31 - 32 weeks of gestational age, posterior placenta, $\mathrm{BPP}=6 / 8($ Activity $=0$ ).

For this patient, jugular catheter (shaldon) was prepared on the next day and she was undergone plasmapheresis $(40 \mathrm{cc} / \mathrm{kg}$ ) as well as gemfibrozil $600 \mathrm{mg}$ BID, Insulin, and Heparin 5000 IU TDS.

After her first plasmapheresis, TG and total cholesterol showed 2626 and 500, respectively, and, then plasmapheresis was done for the patient six times in total and TG and total cholesterol were decreasing. During this time, pregnancy ultrasound, BPP, and AFI were normal and the fetus was monitored closely many times. The patient was discharged after 21 days at 34 weeks of gestational age and with normal blood analysis $(\mathrm{Hb}=11.1, \mathrm{HCT}=33.7, \mathrm{MCV}=94.3$, $\mathrm{MCH}=29.6, \mathrm{PLT}=27000, \mathrm{PT}=10.5, \mathrm{Cr}=0.6, \mathrm{Na}=136, \mathrm{~K}=4.4$, total cholesterol $=191, \mathrm{TG}<500, \mathrm{BS}=89, \mathrm{TSH}=1.41$, Amylase and lipase $=\mathrm{NL}$ ). The patient moved to her city in Kerman province (Bam city) and finally delivered at 37 weeks after 21 days of hospitalization. She is completely healthy at present.

\section{Discussion}

Acute gestational pancreatitis is rarely associated, yet can be caused by important factors, such as maternal mortality and fetal loss. It is generally believed that APIP is associated with several factors (1); gallstone disease or hypertriglyceridemia are the most frequent, and gallstones are amongst top reasons (2). Other reasons, such as Hyperthyroidism (Hyper parathyroidism), connective tissue diseases, infections, and trauma are not the most common causes of acute gestational pancreatitis (3). Acute pancreatitis is explained after medical abortion (4).

Although gallstones are more common causes than hypertriglyceridemia in acute pancreatitis in pregnancy, yet it tends to the severe type in the third trimester (5). Physiological changes in pregnancy, such as overweightness, raised triglycerides, and elevated level of estrogen can increase the incidence of acute pancreatitis in pregnancy (6).

Diagnosis of acute gestational pancreatitis is very difficult, particularly in the first trimester towards the third trimester (6).

Ruptured corpus luteal cyst and ectopic pregnancy are part of differential diagnoses (6). Associations of APIP with HELLP syndrome, preeclampsia, and diabetes mellitus type 2 are important points and can lead to preterm delivery and increase fetal mortality and mobility (7). 


\begin{tabular}{lcccc}
\hline Table 1. Ethiology of Acute Pancreatitis & & & & \\
\hline Mechanical & Toxic & Metabolic & Drugs & Infection \\
\hline Trauma & Congenital & Vascular & Miscellaneous & Genetic \\
\hline
\end{tabular}

Finally, some clinical presentations, namely pain and tenderness in the epigastrium, nausea, vomiting, and abdominal distention led the researchers to characterize pancreatitis; serum amylase and/or lipase were the blood marker for diagnosis. Amylase has severe rise in the first 24 hours and falls down to baseline in three to five days.

Instead, serum lipase remained in the upper normal limit steadily for two weeks. However, there are no differences in both of them for diagnosis $(8,9)$. However, amylase level is not associated with severity (7). The second step is imaging for discovery of the etiology. Abdominal ultrasound and endoscopic ultrasound are useful for diagnosis of APIP with no more additive rise because of Xray radiation (9). Computed tomography, magnetic resonance cholangiopancreatography(MRCP), and endoscopic retrograde cholangiopancreatography (ERCP) are not routine, and should carefully be used case by case (9). Ultimately, the type of pancreatitis should be determined in mild acute pancreatitis (MAP) does not have organ failure and systemic complications recover before long yet in severe acute pancreatitis (SAP), it is presented by permanent organ failure (10).

For APIP, collective bargaining is an expected therapy except in pancreatic abscess or infected effusion or gastrointestinal perforation or the condition deteriorates after active treatment. The best time for surgical intervention is the second trimester that is associated with the least complications (10). Another indication of surgery during pregnancy is the presence of cholangitis (11) that has been identified cholecystectomy during the second trimester, which has no additive risk for the mother and the fetus.

For the current patient after primary study, the researchers understood that amylase $=131$, cholesterol $=1078$, $\mathrm{TG}=6036$ and the ultrasound showed normal gallbladder and liver. As a consequence, the researchers decided to try conservative treatment. Therefore, plasmapheresis (40 $\mathrm{cc} / \mathrm{kg}$ ) was done six times in total and then TG and cholesterol were decreased. Meanwhile, gemfibrozil 600 mg BID, insulin, and Heparin 5000 IU TDS were ordered. With sever acute pancreatitis diagnosis, the researchers started Total Parental Nutrition (TPN) and hospitalized the patient NPO for 21 days. Many studies have shown that in mild acute pancreatitis (MAP), nutritional support is not recommenced and low fat diet can be used in the first week (12).

Advising antibiotics in sever acute pancreatitis is controversial and the researchers did not use it in this case.
There is no more evidence for using antibiotics in mild acute pancreatitis (13).

For the current patient, two other similar cases were reported before by hypertriglyceridemia-induced AP. It is recommended to treat her by plasmapheresis and she was discharged after 21 days from hospitalization in 34 weeks of gestational age and ultimately she delivered at 37 weeks.

\section{Acknowledgments}

The paper was supported by the Department of Obstetrics and Gynecology, Department of Surgery, and Department of Internal Medicine, Ali Ebne Abitaleb Hospital, Zahedan University of Medical Science, Iran.

\section{Footnotes}

Authors' Contribution: Farahnaz Farzaneh examined the patient and wrote the abstract, introduction, and case. Arezoo Esmaeilzadeh wrote the conclusion and Raheleh Rezaei gathered the data.

Conflict of Interests: The authors declare no conflict of interest regarding the publication of this paper.

Funding/Support: The authors declare that the current research was done with no support from any organizations and/or universities.

Patient Consent: It is not declared by the authors.

\section{References}

1. Qihui C, Xiping Z, Xianfeng D. Clinical study on acute pancreatitis in pregnancy in 26 cases. Gastroenterol Res Pract. 2012;2012:271925. doi: 10.1155/2012/271925. [PubMed: 23213326]. [PubMed Central: PMC3506915].

2. Pitchumoni CS, Yegneswaran B. Acute pancreatitis in pregnancy. World J Gastroenterol. 2009;15(45):5641-6. doi: 10.3748/wjg.15.5641. [PubMed: 19960559]. [PubMed Central: PMC2789215].

3. Sreelatha S, Vedavathy N, Nataraj I. Acute pancreatitis in pregnancy. Indian J Clin Prac. 2012;23(4).

4. Eddy JJ, Gideonsen MD, Song JY, Grobman WA, O'Halloran P. Pancreatitis in pregnancy. Obstet Gynecol. 2008;112(5):1075-81. doi: 10.1097/AOG.0b013e318185a032. [PubMed: 18978108]. [PubMed Central: PMC2833089].

5. Zhang DL, Huang Y, Yan L, Phu A, Ran X, Li SS. Thirty-eight cases of acute pancreatitis in pregnancy: A 6-year single center retrospective analysis. J Huazhong Univ Sci Technolog Med Sci. 2013;33(3):361-7. doi: 10.1007/s11596-013-1125-8. [PubMed: 23771661]. 
6. Thulasidass K, Chowdhury TA. Hypertriglyceridemic pancreatitis in pregnancy: Case reports and review of the literature. JRSM Short Rep. 2013;4(8):2.0425333134812E+15. doi:10.1177/2042533313481211. [PubMed: 24040494]. [PubMed Central: PMC3767063].

7. Papadakis EP, Sarigianni M, Mikhailidis DP, Mamopoulos A, Karagiannis V. Acute pancreatitis in pregnancy: An overview. Eur J Obstet Gynecol Reprod Biol. 2011;159(2):261-6. doi: 10.1016/j.ejogrb.2011.07.037. [PubMed: 21840110].

8. Pandey R, Jacob A, Brooks H. Acute pancreatitis in pregnancy: Review of three cases and anaesthetic management. Int J Obstet Anesth. 2012;21(4):360-3. doi: 10.1016/j.ijoa.2012.07.004. [PubMed: 22959261]

9. Banks PA, Bollen TL, Dervenis C, Gooszen HG, Johnson CD, Sarr MG, et al. Classification of acute pancreatitis-2012: Revision of the Atlanta classification and definitions by international consensus. Gut. 2013;62(1):102-11. doi: 10.1136/gutjnl-2012-302779. [PubMed: 23100216].
10. Tang SJ, Rodriguez-Frias E, Singh S, Mayo MJ, Jazrawi SF, Sreenarasimhaiah J, et al. Acute pancreatitis during pregnancy. Clin Gastroenterol Hepatol. 2010;8(1):85-90. doi: 10.1016/j.cgh.2009.08.035. [PubMed: 19747985].

11. Jafri NS, Mahid SS, Idstein SR, Hornung CA, Galandiuk S. Antibiotic prophylaxis is not protective in severe acute pancreatitis: A systematic review and meta-analysis. Am J Surg. 2009;197(6):806-13. doi: 10.1016/j.amjsurg.2008.08.016. [PubMed: 19217608].

12. Igbinosa O, Poddar S, Pitchumoni C. Pregnancy associated pancreatitis revisited. Clin Res Hepatol Gastroenterol. 2013;37(2):177-81. doi 10.1016/j.clinre.2012.07.011. [PubMed: 22959401].

13. Piri F, Firouzkouhi M, Abdollahimohammad A, Mirzaiepour M. Exploring pemphigus challenges based on the patient experiences: A descriptive phenomenological research. La Prensa Medica. 2017;103(6). doi: 10.4172/lpma.1000268. 